\title{
Manejo e Tratamento da Radiodermite em Pacientes Oncológicos: Série de Casos
}

\author{
Management and Treatment of Radiodermatitis in Cancer Patients: Case Series
}

\begin{abstract}
Karine Corcione Turke ${ }^{1}$, Jean Henri Maselli Schoueri ${ }^{1}$, Amanda Voltareli C. Oliveira ${ }^{2}$, Vinicius Lippi ${ }^{2}$, Fernanda Laraia da Rocha Lobo ${ }^{3}$, Julian Gonzalez Fraga ${ }^{4}$, Camila Campos ${ }^{4}$, Vanessa Salzano ${ }^{5}$, Carlos D’Apparecida Machado ${ }^{6}$, Auro del Giglio ${ }^{7}$, Dolores Gonzalez Fabra ${ }^{8}$
\end{abstract}

Com citar: Turke KC, Schoueri JHM, Oliveira AVC. Manejo e Tratamento da Radiodermite em Pacientes Oncológicos: Série de Casos. Clin Onc Let. 2020; Ahead of Print. http://dx.doi.org/10.4322/col.2019.006

\begin{abstract}
Resumo
No presente artigo, apresentamos uma série de três casos de pacientes em acompanhamento oncológico que desenvolveram lesões cutâneas por radioterapia - Radiodermite -, acompanhado de uma revisão integrativa da literatura médica. Apesar de frequente na prática clínica e de apresentar repercussões em ambos, tratamento e qualidade de vida do indivíduo, há poucos casos na literatura sobre o manejo dermatológico de pacientes oncológicos.
\end{abstract}

Palavras-chave: Radiodermite; Tratamento; Relato de caso

\begin{abstract}
In the present article, we present a series of three cases of patients undergoing cancer treatment who developed skin lesions by radiotherapy - Radiodermatitis -, alongside with an integrative review of the medical literature. Despite being frequent in clinical practice and having repercussions on both treatment and quality of life of the individual, there are few cases in the literature on the dermatological management of cancer patients.
\end{abstract}

Keywords: Radiodermatitis; Treatment; Case Report

\section{INTRODUÇÃO}

O câncer é um problema de saúde pública mundial, sendo uma das principais causas de morte em países de qualquer nível econômico. ${ }^{1}$ Há, no entanto, diversas abordagens terapêuticas para a doença, tais como cirurgia, quimio e radioterapia. ${ }^{2}$ Com relação à última, espera-se que $50 \%$ dos pacientes oncológicos sejam submetidos a esse tipo de tratamento ${ }^{3}$ e cerca de $95 \%$ manifestem algum grau de Radiodermite. ${ }^{4}$

De modo geral, a Radiodermite é definida como uma reação inflamatória cutânea resultante da exposição à radiação ionizante. ${ }^{5}$ As manifestações cutâneas relacionadas à radioterapia geralmente aparecem dentro de 1-4 semanas do início da radiação, persistindo pela duração da terapia de radiação e entre 10 e 14 dias pós-tratamento, quando as células basais danificadas migram para a superfície da pele. Isso resulta em inúmeras complicações, como atrasos no tratamento, diminuição do apelo estético e redução da qualidade de vida. ${ }^{4}$

Do conhecimento dos autores, há, na literatura médica, poucos guidelines referentes à abordagem clínica-dermatológica das lesões decorrentes de Radiodermite em pacientes com câncer. Assim, descrevemos

\footnotetext{
${ }^{1}$ Faculdade de Medicina do ABC, Santo André - São Paulo, Brasil

2 Residentes de Dermatologia da Faculdade de Medicina do ABC, Santo André - São Paulo, Brasil

${ }^{3}$ Residente de Oncologia da Faculdade de Medicina do ABC, Santo André - São Paulo, Brasil

${ }^{4}$ Acadêmico de Medicina do Centro Universitário Lusíada, Santos - São Paulo, Brasil

${ }^{5}$ Colaboradora Voluntária no Ambulatório de Reabilitação Dermatológica para Pacientes Oncológicos da Faculdade de Medicina do ABC, Santo André - São Paulo, Brasil

${ }^{6}$ Professor Titular do Departamento de Dermatologia da Faculdade de Medicina do ABC, Santo André - São Paulo, Brasil

${ }^{7}$ Professor Titular do Departamento de Oncologia e Hematologia, Faculdade de Medicina do ABC, Santo André - São Paulo, Brasil

${ }^{8}$ Médica Responsável pelo Ambulatório de Reabilitação Dermatológica para Pacientes Oncológicos da Faculdade de Medicina do ABC, Santo André - São Paulo, Brasil

Financiamento: Não houve financiamento para o presente artigo

Conflito de interesse: Os autores declararam não haver conflitos de interesse que precisam ser informados.
} 
3 relatos de pacientes nos quais o manejo dermatológico mostrou-se bem-sucedido, e revisamos a literatura sobre o tema.

\section{DESCRIÇÃO DOS CASOS}

Na presente série de casos, três pacientes oncológicos, sendo duas mulheres e um homem, com mediana de idade de 69 anos, são descritos. Dentre os pacientes relatados, 66,6\% apresentavam neoplasia maligna de cabeça e pescoço. Todos apresentavam radiodermite associada a infeç̧ão secundária. Deve-se ressaltar que, apesar da indicação inicial de interrupção do tratamento oncológico, todos os pacientes deram continuidade enquanto recebiam tratamento dermatológico devido à melhora apresentada.

\section{CASO 1}

DMS, paciente feminina, 74 anos, apresentou início dos sintomas com odinofagia, tosse, rouquidão e linfonodomegalia cervical à esquerda. À realização de biópsia - em fevereiro de 2016 -, constatou-se tratar de um Carcinoma Espinocelular Moderadamente Diferenciado Superficialmente Invasivo. Iniciou tratamento com quimioterapia (QT) - Carboplatina semanal - concomitante à radioterapia (RT). Ao total, realizou 35 sessões de RT e 6 ciclos de QT. A QT foi suspensa em último atendimento (19/05/17) por toxicidade hematológica, porém optou-se por manter a RT. Evoluiu com Radiodermite grau 4 associada a ardor, febre esporádica e secreção purulenta. Após 7 dias de tratamento composto por antibioticoterapia oral (cefalexina prescrita por 14 dias), antibioticoterapia tópica (rifamicina spray), colutório oral (cloridrato de benzidamina spray prescrito devido à queixa de odinofagia), compressas com água boricada e anti-histamínico (hidroxizine $25 \mathrm{mg} 1 \mathrm{vez}$ por dia), paciente regrediu para Radiodermite Grau I (Figura 1).
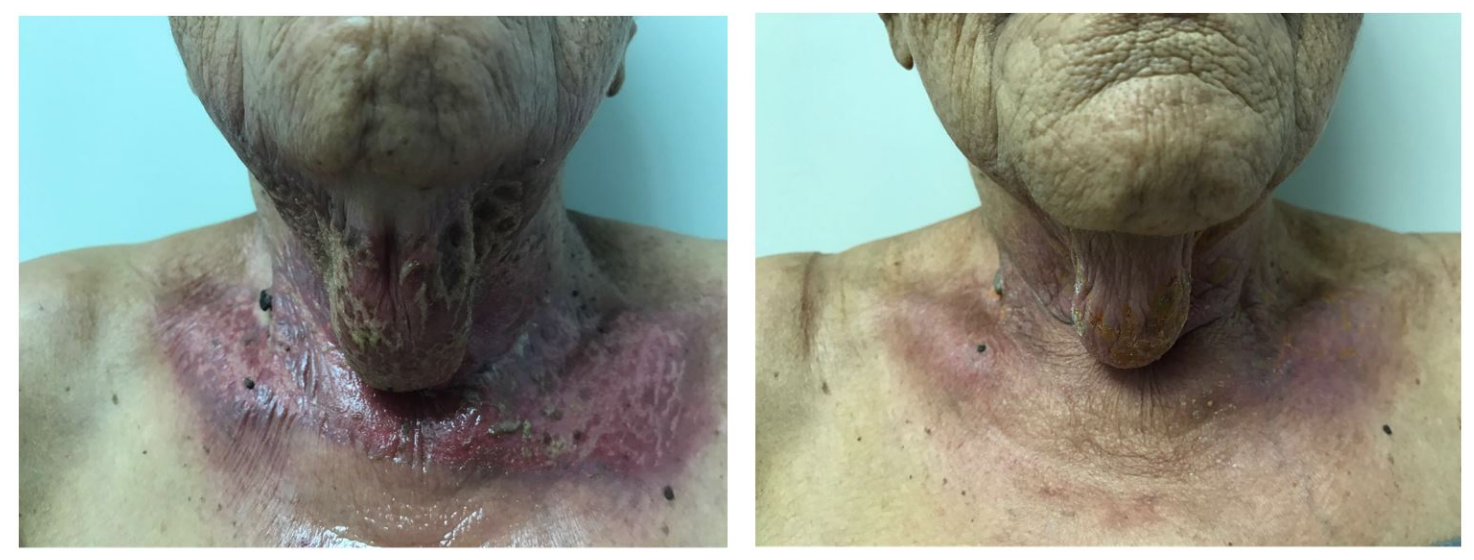

Figura 1. Antes e após 15 dias de tratamento.

\section{CASO 2}

BMA, paciente feminina, 52 anos, diagnosticada com câncer de mama à direita em novembro de 2003. Realizou mastectomia com esvaziamento axilar - margens livres e 4/16 linfonodos positivos - e radioterapia no plastrão cirúrgico e fossa supra-clavicular direita. Submetida a 4 ciclos de quimioterapia adjuvante com esquema AC (Doxorrubicina e Ciclofosfamida) de fevereiro a julho de 2004, quando iniciou tratamento com tamoxifeno - término em julho de 2009.

Devido à recidiva local em novembro de 2016, realizou 4 ciclos de quimioterapia com TC (Ciclofosfamina e Docetaxel), acrescida de radioterapia na topografia de recidiva (22/25 sessões), tendo evoluído com Radiodermite Grave com fistulização em região de quadrante superior direito da transição tóraco-abdominal (Grau 4).

Frente ao quadro, a conduta dermatológica foi a seguinte: antibioticoterapia oral (cefalexina por 10 dias), antibioticoterapia tópica (rifamicina spray - 3 vezes ao dia sobre área de exulceração/fistulização), corticoterapia 
tópica (fuorato de mometasona creme - 2 vezes ao dia) e hidratantes tópicos contendo glicerina e óleo de macadâmia - 2 vezes ao dia.

Iniciou Anastrozol em maio de 2017. Atualmente, em acompanhamento e em uso da medicação (Figura 2).
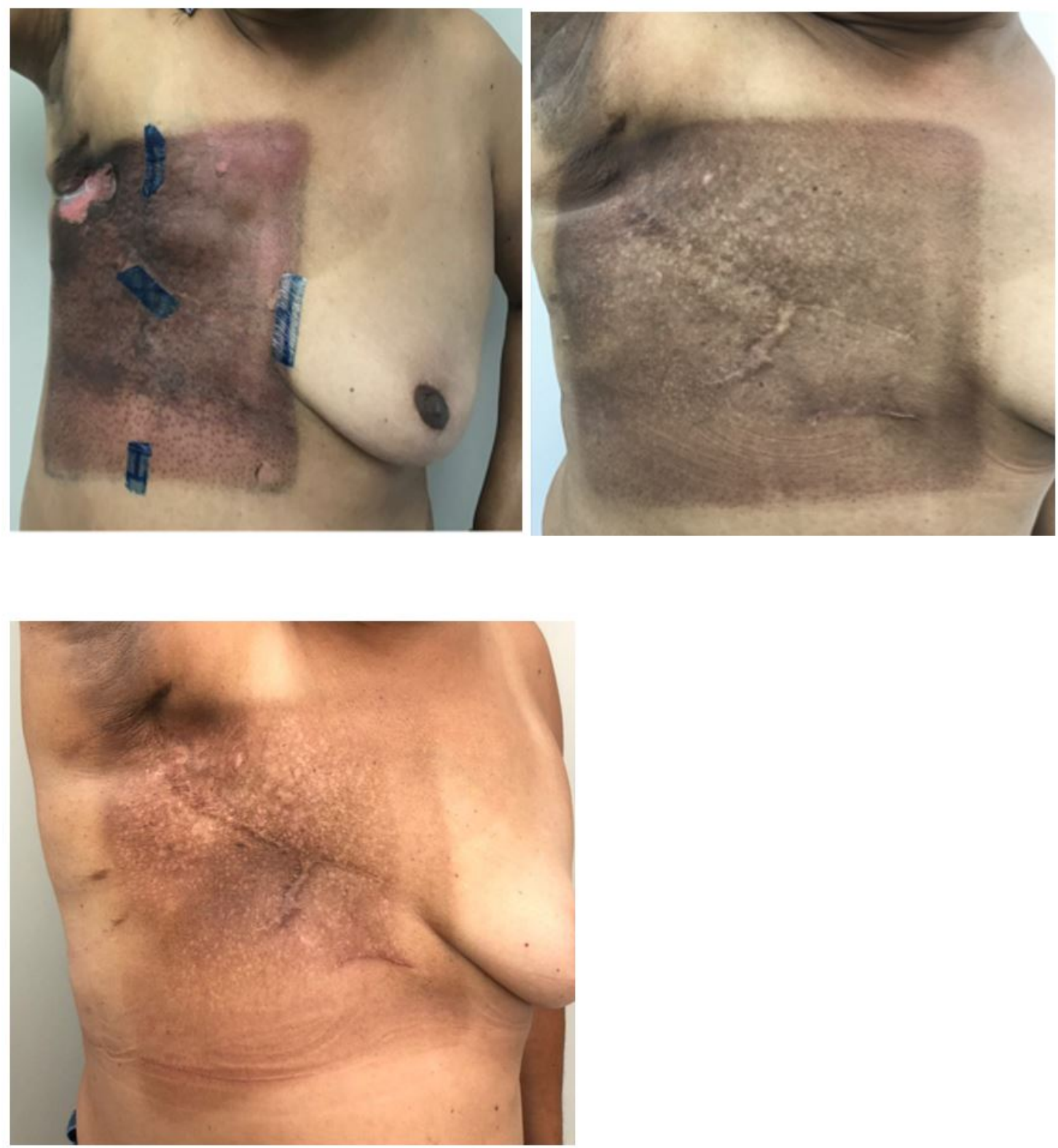

Figura 2. Antes e depois do tratamento dermatológico. Na primeira consulta, a paciente apresentava-se com fita adesiva para proteção da marcação em cima da lesão, bem como um fístula infra-axilar, que lhe causava intensa dor. Com a instituição do tratamento dermatológico e orientações adequadas, houve regressão da dor em 03 dias e da fístula, em 15 dias. A última foto apresentada (resultado final) foi tirada após 40 dias de tratamento.

\section{CASO 3}

JDL, paciente masculino, 69 anos, com diagnóstico de câncer de laringe T2N2 em dezembro de 2016. Foi submetido a tratamento com Cetuximabe - 20 sessões (programado 23 sessões) e RT 3 sessões (programado 16 sessões). Por apresentar múltiplas pústulas sobre base eritematosa - algumas coalescentes - em tronco anterior e posterior, e placa eritematosa com crostas melicéricas em região cervical anterior, juntamente com limitação de 
movimento e rouquidão há 10 dias, teve o tratamento oncológico interrompido. Foi iniciado antibióticoterapia oral (cefalexina - 7 dias) e tópica (eritromicina 2 vezes ao dia), corticoterapia tópica (fuorato de mometasona creme 2 vezes ao dia) e hidratação tópica contendo glicerina e óleo de macadâmia - 2 vezes ao dia. Após 7 dias, paciente evoluiu com regressão importante das lesões com melhora significativa do eritema inicial junto com diminuição das pústulas em região cervical e dorso (Figura 3).
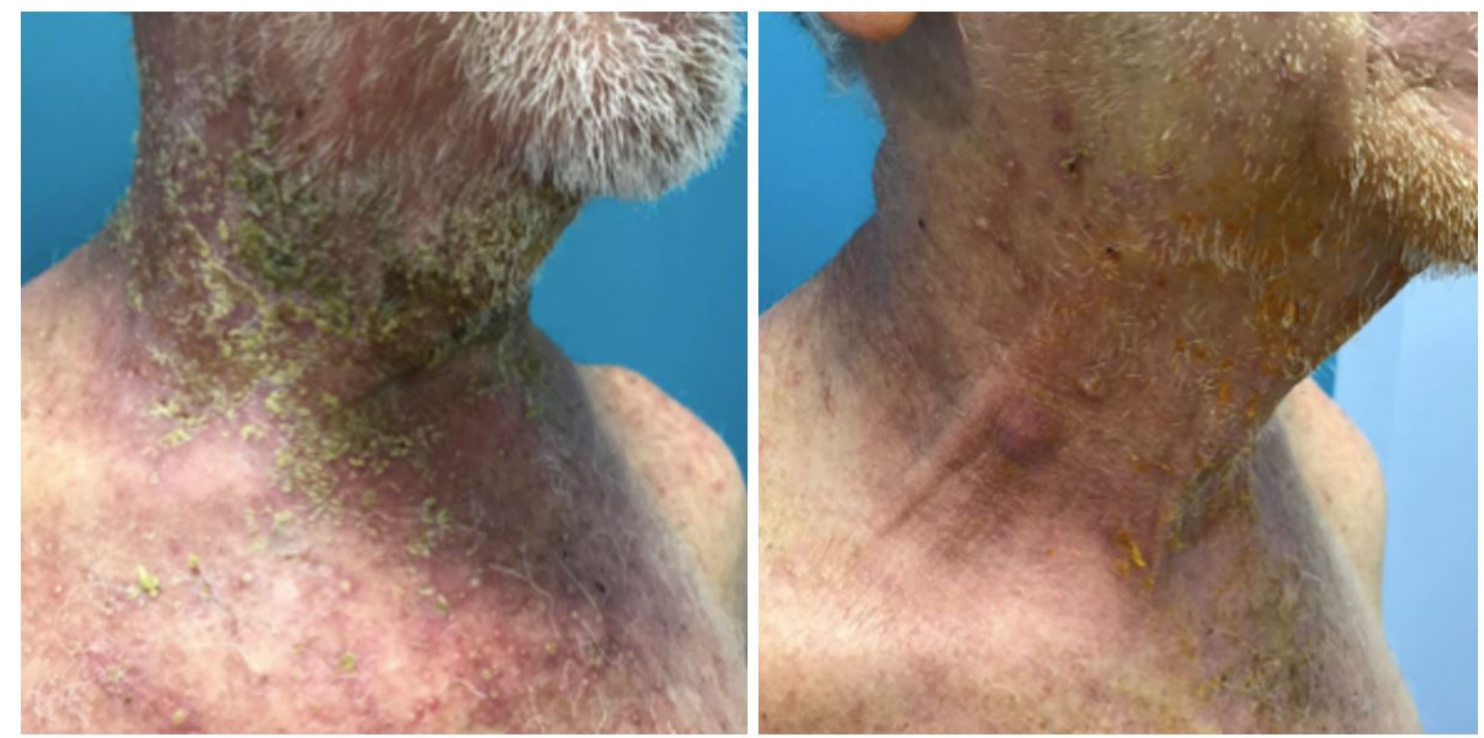

Figura 3. Antes e após 15 dias de tratamento dermatológico. Não houve interrupção do tratamento oncológico.

\section{REVISÃO DA LITERATURA}

\section{I) PANORAMA GERAL}

A radioterapia, utilizada de forma isolada ou combinada a outros tratamentos antineoplásicos é uma ferramenta importante para o tratamento de cânceres primários. ${ }^{6}$ Desde seu surgimento, obteve-se grande ganho no controle local das doenças a longo prazo, bem como na sobrevida. ${ }^{7}$ No entanto, efeitos agudos e crônicos relacionados a sua administração aos poucos foram categorizados.

Apesar da pele não ser um órgão que limita as doses administradas de radioterapia, a toxicidade cutânea prejudica a qualidade de vida devido à dor, desconforto e limitação das atividades diárias ${ }^{8}$. A radiodermite em câncer de mama é um dos objetos de estudo mais encontrados na literatura. ${ }^{7,9-11}$ Tal constatação provavelmente deve-se a estatística abundante de que mais de $90 \%$ das mulheres submetidas a este tratamento desenvolvem toxicidade cutânea o que pode, inclusive, limitar o tempo total previsto para a terapêutica, comprometendo o controle da doença. ${ }^{10}$ Logo, o principal desafio da equipe de saúde é minimizar os efeitos colaterais sem que isso limite a eficácia do tratamento ${ }^{7}$

Apesar de diversos estudos abordarem tratamentos e profilaxias para radiodermites, há ainda grande heterogeneidade na prática clinica a depender de cada instituição.

As ferramentas mais usadas para graduar os efeitos relacionados são:

- Critérios Terminológicos Comuns para Efeitos Adversos (CTCEA) versão 4.0,

- Grupo Oncológico de Radioterapia (RTOG)/ Organização Europeia de Pesquisa

- Efeito tardio em tecidos normais (LENT)/Medidas Objetivas para os sintomas e cuidados (SOMA).

Essas escalas reúnem vários efeitos adversos experimentados por pacientes durante o tratamento e atribuem um grau de severidade para cada categoria. Apesar de amplamente utilizadas, há poucas evidências científicas que validem essas ferramentas, o que faz com que o ideal seja utilizá-las em conjunto. ${ }^{12,13}$ (Tabela 1 ) 
Tabela 1. Tabela referente às classificações usadas na radiodermite aguda, reproduzida, na íntegra, de Wong et al. ${ }^{14}$

\begin{tabular}{|c|c|c|c|}
\hline & RTOG & LENT/SOMA & CTCAE 4.0 \\
\hline 0 & $\begin{array}{l}\text { Nenhuma alteração em relação à } \\
\text { linha de base / sem sintomas }\end{array}$ & $\begin{array}{l}\text { Nenhuma alteração em relação à } \\
\text { linha de base / sem sintomas }\end{array}$ & $\begin{array}{c}\text { Nenhuma alteração em relação à } \\
\text { linha de base / sem sintomas }\end{array}$ \\
\hline 1 & $\begin{array}{c}\text { Eritema folicular, fraco ou sem brilho, } \\
\text { epilação, descamação seca, sudorese } \\
\text { diminuída }\end{array}$ & $\begin{array}{c}\text { Sintomas menores que não requerem } \\
\text { tratamento }\end{array}$ & Eritema fraco ou descamação seca \\
\hline 2 & $\begin{array}{l}\text { Eritema macio ou brilhante, descamação } \\
\text { úmida irregular, edema moderado }\end{array}$ & $\begin{array}{l}\text { Sintomas moderados que requerem } \\
\text { tratamento conservador }\end{array}$ & $\begin{array}{l}\text { Eritema moderado a vigoroso, } \\
\text { descamação úmida irregular, } \\
\text { confinada principalmente a dobras e } \\
\text { pregas da pele, edema moderado }\end{array}$ \\
\hline 3 & $\begin{array}{c}\text { Descamação úmida e confluente, } \\
\text { exceto dobras cutâneas, edema } \\
\text { depressível }\end{array}$ & $\begin{array}{l}\text { Sintomas graves, que têm um } \\
\text { impacto negativo significativo nas } \\
\text { atividades diárias e requerem } \\
\text { tratamento mais agressivo }\end{array}$ & $\begin{array}{c}\text { Descamação úmida que não seja em } \\
\text { dobras e pregas da pele, sangramento } \\
\text { induzido por pequenos traumas ou } \\
\text { abrasão }\end{array}$ \\
\hline 4 & Ulceração, necrose hemorrágica & $\begin{array}{l}\text { Dano funcional irreversível, } \\
\text { necessitando de grande intervenção } \\
\text { terapêutica }\end{array}$ & $\begin{array}{l}\text { Consequências com risco de vida, } \\
\text { necrose da pele ou ulceração da derme } \\
\text { de espessura total, sangramento } \\
\text { espontâneo do local envolvido, } \\
\text { indicação de enxerto de pele }\end{array}$ \\
\hline 5 & $\begin{array}{c}\text { Morte relacionada aos efeitos do } \\
\text { tratamento }\end{array}$ & Morte ou perda de órgão & Morte \\
\hline
\end{tabular}

\section{II) FISIOPATOGENIA}

A pele é uma membrana isolante que apresenta diversas propriedades, como impermeabilidade relativa à água e eletrólitos; resistência a agentes danosos corrosivos; alta impedância elétrica, superfície relativamente seca (evitando a proliferação de microorganismos). ${ }^{15}$ Apesar de todas essas características, configura-se como órgão vulnerável às lesões causadas pela radiação, já que prolifera-se rapidamente e está em processo de maturação celular contínuo. ${ }^{6} \mathrm{O}$ mecanismo das radiodermites não é completamente conhecido, porém sabe-se que a radiação ionizante induz quebras no DNA e geram espécies reativas de oxigênio, levando à injúria e morte celular, além de promover à inflamação. Os danos diretos ocorrem na epiderme, folículos pilosos (especialmente em células tronco), estruturas anexiais, fibroblastos e células endoteliais. Na pele normal, a epiderme e o estrato córneo atuam como uma barreira para a perda de água e com isso mantêm a hidratação cutânea. O dano causado pela radioterapia na epiderme e estruturas anexiais pode levar à perda da função de impermeabilidade, resultando em perda de hidratação; comprometimento da manutenção do pH ácido e alteração da secreção sebácea. Além disso, pode haver indução de uma resposta inflamatória com vasodilatação, o que leva a um aumento do fluxo sangüíneo seguido por hiperpigmentação pós-inflamatória. A radiação pode comprometer todos os componentes atribuídos à integridade cutânea e à hidratação do estrato córneo por meio da indução de apoptose ou necrose, redução da síntese de lipídeos e defeitos nas estruturas de adesão celular. ${ }^{6}$

$\mathrm{O}$ pH normal da pele varia entre 4 e 7 . $\mathrm{O}$ aumento do pH após a radiação reflete o comprometimento de estruturas anexiais que mantêm o mesmo. Portanto, a alcalinização pode reduzir a função de barreira, já que as enzimas responsáveis pela permeabilidade requerem $\mathrm{pH}$ ácido para manterem-se ativas. Como consequência predispõe-se o crescimento de microrganismos como Candida albicans e Stafilococcus aureus que crescem melhor em pH neutro, levando ao comprometimento da cicatrização.

Pode haver ainda uma "up regulation" na expressão de citocinas angiogênicas (VEGF) levando à formação de telangectasias. Com isso, há uma redução da hidratação cutânea, aumento do $\mathrm{pH}$, da pigmentação e do fluxo sanguíneo, porém sem alteração do nível sebáceo. ${ }^{6}$

\section{III) CLASSIFICAÇÃO}

O dano cutâneo pode ser classificado em agudo ou crônico. Os efeitos agudos ocorrem horas a semanas após a exposição e constituem em eritema, edema, descamação seca e úmida, discromias, despilificação ou até mesmo danos severos como ulceração. Já as formas crônicas, surgem meses a anos após a exposição e apresentam-se como 
úlceras tardias, fibrose e telangectasias. ${ }^{12,16}$ Alguns autores consideram as complicações de fase aguda incidentes entre 2 a 3 semanas após o tratamento e as tardias após 90 dias do tratamento. Enquanto a maioria dos eventos agudos curam-se de forma espontânea, ou no caso dos sintomáticos dentro de 2 a 3 semanas, os tardios tendem a persistir e causar efeito negativo na qualidade de vida dos pacientes ${ }^{12,14,17}$

A severidade do dano cutâneo após a radiação é influenciada pelo tratamento e pelos fatores relacionados ao paciente, que são:6,13

- Desnutrição

- Dano actínico

- Obesidade

- Doenças do tecido conectivo (lúpus eritematoso, esclerodermia)

- Fatores genéticos

Por sua vez, os fatores relacionados à radioterapia que podem afetar a severidade da dermatite são:

- Dose total da irradiação

- Sítio irradiado

- Tempo de fracionamento

- Volume dos tecidos irradiados

- Área da superfície exposta

Além disso, a técnica de radiação também influencia na severidade da radiodermite. A radioterapia modulada por intensidade (IMRT) mostrou ser superior em relação à irradiação convencional de mama total baseada em cunha no que diz respeito à redução dos sintomas, fornecendo uma dose mais homogênea através da mama e removendo os pontos quentes de radiação. ${ }^{7}$

Pignol et al. ${ }^{18}$ realizaram um ensaio clinico randomizado multicêntrico, duplo cego com 331 pacientes que realizaram radioterapia adjuvante de mama distribuídos de forma aleatória em 2 grupos; tratados com radioterapia padrão e IMRT que foram avaliados a cada semana durante o tratamento e até 6 semanas após o término. A incidência de descamação úmida ocorreu em $31,2 \%$ do grupo tratado com IMRT e $47,8 \%$ com tratamento padrão $(P<0,001)$. Uma análise multivariada descobriu que o uso de IMRT mamário $(P=0,003)$ e menor tamanho da mama $(P<0,001)$ foram significativamente associados a um menor risco de descamação úmida. $O$ uso de IMRT não se correlacionou com a dor e a qualidade de vida. A presença de descamação úmida correlacionou-se significativamente com a dor $(P=0,002)$ e uma qualidade de vida reduzida $(P=0,003)$.

Um outro estudo conduzido por Freedman et al. ${ }^{19}$ descrevem que a Radioterapia Modulada por Intensidade (IMRT) leva à uma forma de dermatite menos grave, bem como a lesões que necessitam de menos tempo para serem tratadas, se comparadas à radioterapia convencional.

Além da radiodermite, a radioterapia causa toxicidades agudas e tardias que afetam vários órgãos e funções. Uma das toxicidades agudas mais comuns é a mucosite, devido à irradiação da mucosa. No caso de toxicidade tardia, a característica mais comum é a xerostomia, onde a diminuição considerável da saliva leva à secura persistente da boca, desconforto oral, dor de garganta, deterioração dentária, dificuldade de fala, alteração do sabor e comprometimento das funções de mastigação e de deglutição, que podem levar à depleção nutricional e perda de peso. ${ }^{20}$ De acordo com os resultados publicados, a técnica IMRT melhora os perfis de toxicidade sem comprometer a eficácia. ${ }^{21-23}$

Com relação à toxicidade tardia, estudos comparativos mostram diferenças entre as técnicas convencionais RT e IMRT com relação à xerostomia avaliada nos pacientes, ${ }^{21,22,24,25}$ que demonstram um grau significativamente menor de xerostomia quando tratados com técnica IMRT do que aqueles tratados com técnicas convencionais de radioterapia.

A explicação para esta toxicidade menor relacionada ao IMRT é que a preservação da função das glândulas salivares tem um efeito protetor em relação à toxicidade oral induzida por radiação e às infecções bucais secundárias. ${ }^{26}$ As doses médias de radiação da glândula parótida para pacientes tratados com IMRT foram 
significativamente menores em comparação com as doses médias de radiação da glândulas parótidas de pacientes tratados com 2-3D RT (Mann Whitney, $P=0,016$ ). Foi encontrada correlação significativa entre xerostomia tardia e dose média de radiação da glândula parótida (teste de spearman, rho $=0,5013, P<0,001$ ). Numerosos estudos também relataram correlação significativa entre a dose média de radiação da parótida e o fluxo salivar após a RT com a taxa de pacientes que sofreram de xerostomia. ${ }^{23,27}$

Kouloulias et al ${ }^{20}$ realizaram uma revisão sistemática de resultados de toxicidade aguda e tardia coletados de 33 estudos comparativos entre pacientes tratados com técnicas de RT convencionais (2DRT e 3DCRT) e aqueles tratados com técnica IMRT para carcinomas de cabeça e pescoço. A principal conclusão do estudo foi que o IMRT reduz a xerostomia tardia quando comparada com ambas, a radioterapia convencional tridimensional (3D-CRT) e a radioterapia convencional de duas dimensões (2DRT). Também há tendência de superioridade ao uso IMRT no que diz respeito à redução da mucosite aguda.

A radiodermite acarreta prejuízos significativos na qualidade de vida dos pacientes oncológicos, sendo necessárias intervenções multiprofissionais e novos estudos voltados para a prevenção e tratamento da lesão. ${ }^{28}$

\section{COMENTÁRIO}

Frente ao grande número de informações apresentadas, faz-se necessário colocar alguns pontos em destaque. Primeiro, apesar das evidências dos estudos mostrarem que a técnica IMRT acarreta menor número de casos de radiodermite quando comparado a técnicas convencionais de radioterapia, a técnica convencional tem-se mostrado como a realidade em nosso serviço, já que poucos pacientes no Brasil têm acesso à IMTR. ${ }^{29}$

Segundo, nossa experiência mostra que o uso de Fuorato de Mometasona parece ser eficaz no manejo de radiodermites com lesões secas; já nos casos de radiodermites com lesões exsulceradas e presença de secreção, o uso de Rifamicina spray associado à Corticoterapia de uso tópico apresentou rápida resolução dos quadros.

Por fim, gostaríamos de salientar um ponto inusitado, porém recorrente em nossa prática clínica: o uso de compressas com chá de camomila em lesões de radiodermite. Por mais que se mostrem eficazes no combate à inflamação cutânea - e que esse conhecimento encontre-se difundido e enraizado em grande parte da população brasileira -, deve-se considerar que a erva do chá pode estar contaminada por fungos e/ou bactérias que acarretariam graves infecções cutâneas. Nesse sentido, optamos por contraindicar o seu uso. Ainda, caso haja monilíase associada à lesão por radiodermite, o uso de cremes com associação de antibiótico e antifúngico têm mostrado excelentes resultados.

\section{CONCLUSÃO}

Na nossa experiência, a prevenção, manejo e tratamento adequado das lesões dermatológicas decorrentes do tratamento oncológico são de suma importância para reduzir os danos causados pela radioterapia e permitir que os pacientes continuem o tratamento oncológico proposto, sem interrupção ou atraso.

O impacto dermatológico da radioterapia gera dor, aumento do sofrimento e piora da qualidade de vida. Ao nosso ver, a presença do dermatologista na equipe multidisciplinar que acolhe o paciente oncológico é fundamental. Entretanto, mais estudos são necessários para comprovar os resultados encontrados.

\section{REFERÊNCIAS}

1. Siegel RL, Miller KD, Jemal A. Cancer statistics, 2016. CA Cancer J Clin. 2016;66(1):7-30. http://dx.doi.org/10.3322/caac.21332. PMid:26742998.

2. Sudhakar A. History of cancer, ancient and modern treatment methods. J Cancer Sci Ther. 2009;1(2):1-4. http://dx.doi.org/10.4172/1948-5956.100000e2. PMid:20740081.

3. Baskar R, Lee KA, Yeo R, Yeoh KW. Cancer and radiation therapy: current advances and future directions. Int J Med Sci. 2012;9(3):193-9. http://dx.doi.org/10.7150/ijms.3635. PMid:22408567. 
4. Singh M, Alavi A, Wong R, Akita S. Radiodermatitis: a review of our current understanding. Am J Clin Dermatol. 2016;17(3):277-92. http://dx.doi.org/10.1007/s40257-016-0186-4. PMid:27021652.

5. National Center for Biotechnology Information. Mesh definition [Internet]. Bethesda: NCBI; 2017 [cited 2017 Aug 24 ]. Available from: https://www.ncbi.nlm.nih.gov/mesh/68011855

6. Hu SC, Hou MF, Luo KH, et al. Changes in biophysical properties of the skin following radiotherapy for breast cancer. J Dermatol. 2014;41(12):1087-94. http://dx.doi.org/10.1111/1346-8138.12669. PMid:25354814.

7. De Langhe S, Mulliez T, Veldeman L, et al. Factors modifying the risk for developing acute skin toxicity after wholebreast intensity modulated radiotherapy. BMC Cancer. 2014;14(1):711. http://dx.doi.org/10.1186/1471-2407-14-711.

8. Fabra DG, Salzano V, Guglielnino F, Kayo AP. Impacto do ambulatório de reabilitação dermatocosmiátrica na qualidade de vida dos pacientes oncológicos da Faculdade de Medicina do ABC. Arq Bras Ciên Saúde, Santo André. 2010;35(2):8994. http://dx.doi.org/10.7322/abcs.v35i2.92.

9. Kraus-Tiefenbacher U, Sfintizky A, Welzel G, et al. Factors of influence on acute skin toxicity of breast cancer patients treated with standard three-dimensional conformal radiotherapy (3D-CRT) after breast conserving surgery (BCS). Radiat Oncol. 2012;7(1):217. http://dx.doi.org/10.1186/1748-717X-7-217. PMid:23249653.

10. Bazire L, Fromantin I, Diallo A, et al. Hydrosorb ${ }^{\circledR}$ versus control (water based spray) in the management of radioinduced skin toxicity: Results of multicentre controlled randomized trial. Radiother Oncol. 2015;117(2):229-33. http://dx.doi.org/10.1016/j.radonc.2015.08.028. PMid:26328937.

11. Heyer E, Smyth W, Laffin N, Gardner A. Protocol paper: a randomized trial of 2 creams in the skin care of patients receiving radiation therapy for breast cancer in the tropics. Cancer Nurs. 2015;38(4):E35-41. http://dx.doi.org/10.1097/NCC.0000000000000175. PMid:25075645.

12. Devlin JW, Skrobik Y, Gélinas C, et al. Clinical practice guidelines for the prevention and management of pain, agitation/sedation, delirium, immobility, and sleep disruption in adult patients in the ICU. Crit Care Med. 2018;46(9):e82573. http://dx.doi.org/10.1097/CCM.0000000000003299. PMid:30113379.

13. Franco P, Potenza I, Moretto F, et al. Hypericum perforatum and neem oil for the management of acute skin toxicity in head and neck cancer patients undergoing radiation or chemo-radiation: a single-arm prospective observational study. Radiat Oncol. 2014;9(1):297. http://dx.doi.org/10.1186/s13014-014-0297-0. PMid:25544371.

14. Wong RKS, Bensadoun RJ, Boers-Doets CB, et al. Clinical practice guidelines for the prevention and treatment of acute and late radiation reactions from the MASCC Skin Toxicity Study Group. Support Care Cancer. 2013;21(10):2933-48. PMid:23942595.

15. Sampaio SAP, Castro RM, Rivitti EA. Dermatologia básica. 3. ed. São Paulo: Artes Médicas; 1983.

16. Pires AMT, Araujo SR, Segreto HRC. Avaliação das reações agudas da pele e seus fatores de risco em pacientes com câncer de mama submetidas à radioterapia. Rev Lat Am Enfermagem. 2008;16(5):844-9.

http://dx.doi.org/10.1590/S0104-11692008000500008.

17. Gieringer M, Gosepath J, Naim R. Radiotherapy and wound healing: principles, management and prospects. Oncol Rep. 2011;26(2):299-307. http://dx.doi.org/10.3892/or.2011.1319. PMid:21617873.

18. Pignol JP, Olivotto I, Rakovitch E, et al. A multicenter randomized trial of breast intensity-modulated radiation therapy to reduce acute radiation dermatitis. J Clin Oncol. 2008;26(13):2085-92. http://dx.doi.org/10.1200/JCO.2007.15.2488. PMid:18285602.

19. Freedman GM, Li T, Nicolaou N, Chen Y, Ma CC-M, Anderson PR. Breast intensity-modulated radiation therapy reduces time spent with acute dermatitis for women of all breast sizes during radiation. Int J Radiat Oncol Biol Phys.

2009;74(3):689-94. http://dx.doi.org/10.1016/j.jjrobp.2008.08.071. PMid:19362779.

20. Kouloulias V, Thalassinou S, Platoni K, et al. The treatment outcome and radiation-induced toxicity for patients with head and neck carcinoma in the IMRT era: a systematic review with dosimetric and clinical parameters. BioMed Res Int. 2013;2013:401261. http://dx.doi.org/10.1155/2013/401261. PMid:24228247. 
21. Clavel S, Nguyen DH, Fortin B, et al. Simultaneous integrated boost using intensity-modulated radiotherapy compared with conventional radiotherapy in patients treated with concurrent carboplatin and 5-fluorouracil for locally advanced oropharyngeal carcinoma. Int J Radiat Oncol Biol Phys. 2012;82(2):582-9. http://dx.doi.org/10.1016/j.ijrobp.2010.10.061. PMid:21277695.

22. Nutting CM, Morden JP, Harrington KJ, et al. Parotid-sparing intensity modulated versus conventional radiotherapy in head and neck cancer (PARSPORT): a phase 3 multicentre randomised controlled trial. Lancet Oncol. 2011;12(2):127-36. http://dx.doi.org/10.1016/S1470-2045(10)70290-4. PMid:21236730.

23. Chao KSC, Deasy JO, Markman J, et al. A prospective study of salivary function sparing in patients with head-and-neck cancers receiving intensity-modulated or three-dimensional radiation therapy: initial results. Int J Radiat Oncol Biol Phys. 2001;49(4):907-16. http://dx.doi.org/10.1016/S0360-3016(00)01441-3. PMid:11240231.

24. Lee NY, Arruda FF, Puri DR, et al. A comparison of intensity-modulated radiation therapy and concomitant boost radiotherapy in the setting of concurrent chemotherapy for locally advanced oropharyngeal carcinoma. Int J Radiat Oncol Biol Phys. 2006;66(4):966-74. http://dx.doi.org/10.1016/j.ijrobp.2006.06.040. PMid:17145527.

25. Lambrecht M, Nevens D, Nuyts S. Intensity-modulated radiotherapy versus parotid-sparing 3D conformal radiotherapy: effect on outcome and toxicity in locally advanced head and neck cancer. Strahlenther Onkol. 2013;189(3):223-9. http://dx.doi.org/10.1007/s00066-012-0289-7. PMid:23319256.

26. Vissink A, Burlage FR, Spijkervet FKL, Jansma J, Coppes RP. Prevention and treatment of the consequences of head and neck radiotherapy. Crit Rev Oral Biol Med. 2003;14(3):213-25. http://dx.doi.org/10.1177/154411130301400306.

PMid:12799324.

27. Maes A, Weltens $C$, Flamen $P$, et al. Preservation of parotid function with uncomplicated conformal radiotherapy. Radiother Oncol. 2002;63(2):203-11. http://dx.doi.org/10.1016/S0167-8140(02)00013-0. PMid:12063010.

28. Rocha DM, Pedrosa AO, Oliveira AC, Bezerra SMG, Benício CDAV, Nogueira LT. Evidências científicas sobre os fatores associados à qualidade de vida de pacientes com radiodermatite. Rev Gaúcha Enferm. 2018;39:e2017-0224. http://dx.doi.org/10.1590/1983-1447.2018.2017-0224.

29. Oliveira HF, Trevisan FA, Bighetti VM, et . Radioterapia de intensidade modulada (IMRT) para pacientes do SUS: análise de 508 tratamentos em dois anos de instalação da técnica. Radiol Bras. 2014;47(6):355-60. http://dx.doi.org/10.1590/0100-3984.2013.1905. PMid:25741118. 